\title{
On the principal centers of a triangle
}

\author{
Michael S. Longuet-Higgins \\ The author is a Senior Research Physicist at the University of California, San Diego. \\ Born in England in 1925, he graduated in mathematics from the University of Cam- \\ bridge in 1946, with a Ph.D. in geophysics in 1951. He has maintained an interest \\ both in pure mathematics (projective geometry, polytopes, random functions and \\ surfaces) and in applied mathematics (fluid dynamics, microseisms, the generation \\ of ocean waves by wind, the dynamics of bubbles, sonoluminescence, and other \\ topics). From 1969 to 1989 he served as a Royal Society Research Professor at the \\ University of Cambridge. His recreations include music, gardening and educational \\ toys. He is married, with 4 children and 8 grandchildren.
}

Eight of the simplest triangle centers, namely $H, I, J, L, M, N, O$ and $I^{\prime}$ (in the present notation) are shown to form a simple pattern of collinearities, which is completed by the addition of a ninth point $K$, a less well-known triangle center; see Figure 3.

Four of the well-known centers on the Euler line, namely $L, O, M$ and $O^{\prime}$, are each a point of concurrence of four lines, each line containing one of the tritangent centers $I_{A}$, $I_{B}, I_{C}$ and $I$ and one other well-known triangle center. The sixteen other centers are: the Feuerbach points $F_{A}, F_{B}, F_{C}$ and $F$; the Gergonne points $G_{A}, G_{B}, G_{C}$ and $G$; the Nagel points $N_{A}, N_{B}, N_{C}$ and $N$; and the countercenters $J_{A}, J_{B}, J_{C}$ and $J$.

\section{Introduction}

A comprehensive publication by Kimberling [3] has listed many of the principal centers of a triangle $A B C$ as well as the various collinearities among them. Most were discovered by accurate computer "drawing". Some but not all have received verification by formal proof.

Die nachfolgende Arbeit ist ein Beitrag zur Elementargeometrie, genauer zur Dreiecksgeometrie. Bekanntlich liegen die Schnittpunkte der Höhenlinien, der Seitenhalbierenden und der Mittelsenkrechten eines Dreiecks auf einer Geraden, der Euler-Geraden. Die Verbindung des Schnittpunkts der Winkelhalbierenden und des Schwerpunkts führt auf die sogenannte Nagel-Gerade, auf der weitere besondere Punkte des Dreiecks liegen. Der Autor stellt hier einen neuen interessanten Punkt vor, der die bekannten neun besonderen Punkte eines Dreiecks in harmonischer Weise zu einem regelmässigen $\mathrm{Mu}$ ster ergänzt. $j k$ 
In the present paper it will be shown that eight of the most elementary centers fall into a group which when augmented by the addition of one further point, not listed by Kimberling [3], form a remarkable family as shown schematically in Figure 3. Here

$O$ is the circumcenter (meet of the perpendicular bisectors of the three sides of $A B C$ ),

$I$ is the incenter (meet of the bisectors of the three angles),

$M$ is the median point (meet of the three medians, each joining a vertex, say $A$, to the mid-point $A^{\prime}$ of the opposite side $B C$ ),

$H$ is the orthocenter (meet of the three altitudes),

$L$ is the Longchamps point (reflection of $H$ in $O$ ),

$N$ is the Nagel point (meet of the three lines $A X_{A}, B Y_{B}, C Z_{C}$, where $X_{A}$ is the point of contact of the excircle opposite $A$ with the side $B C$ ),

$J$ is the Yff point or countercenter (meet of the perpendiculars from the three excenters $I_{A}, I_{B}, I_{C}$ to the corresponding sides of $A B C$ ).

Also

$I^{\prime}$ is the Spieker point (incenter of the median triangle $A^{\prime} B^{\prime} C^{\prime}$ ),

$J^{\prime}$ is the countercenter of $A^{\prime} B^{\prime} C^{\prime}$.

The new point is $K$. It may be defined by construction as the meet of $N H$ and LI. M is here shown to be the median point of the triangle $K L N$, so that $K$ also lies on $J M$. Other properties of $K$ remain to be explored.

In addition to this family of centers which we may call rational because of the ratios of parallel segments, we also discuss the relationship of the Gergonne point $G$, and other associated points, to the scheme of Figure 3. In addition we show that each of the points $L O M O^{\prime}$ on the Euler line is itself a 4 -fold point of concurrence of lines joining other centers of the triangle $A B C$.

\section{The Euler and Nagel lines}

As is well-known, the points $H, M, O$ and $L$ all lie (with many other centers) on the Euler line of the triangle $A B C$, while the points $I, M, I^{\prime}$ and $N$ all lie on a second line, which after Hofstadter [2] we may call the Nagel line. The Euler line HMOL and the Nagel line $I M I^{\prime} N$ thus intersect in the median point $M$, as shown in Figure 1. Moreover, the corresponding segments of these two lines are in proportion:

$$
\left.\begin{array}{r}
H M: M O: O L=2: 1: 3 \\
I M: M I^{\prime}: I^{\prime} N=2: 1: 3
\end{array}\right\}
$$

from which it follows that $H I, O I^{\prime}$ and $L N$ are all parallel and

$$
H I=2 O I^{\prime}=\frac{1}{2} L N .
$$




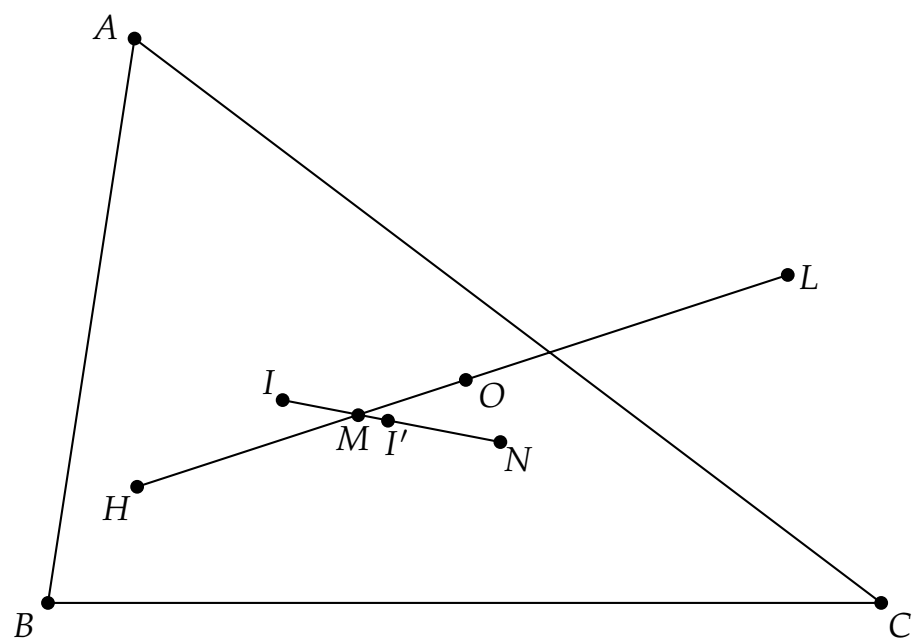

Fig. 1

\section{The countercenter $J$}

In the present note we are specially interested in another center listed by Kimberling [3] which has received no name, though certainly it is one of the simplest centers and, as we shall see, is intimately related to those just mentioned. For reasons that will appear, we name this point the countercenter and denote it by the letter $J$.

$J$ may be defined as the point of concurrence of the three perpendiculars, each from one of the excenters $I_{A}, I_{B}, I_{C}$ to the corresponding side of $A B C$. In Kimberling's [3] notation it is $X_{40}$. Apparently it was discovered by Peter Yff.

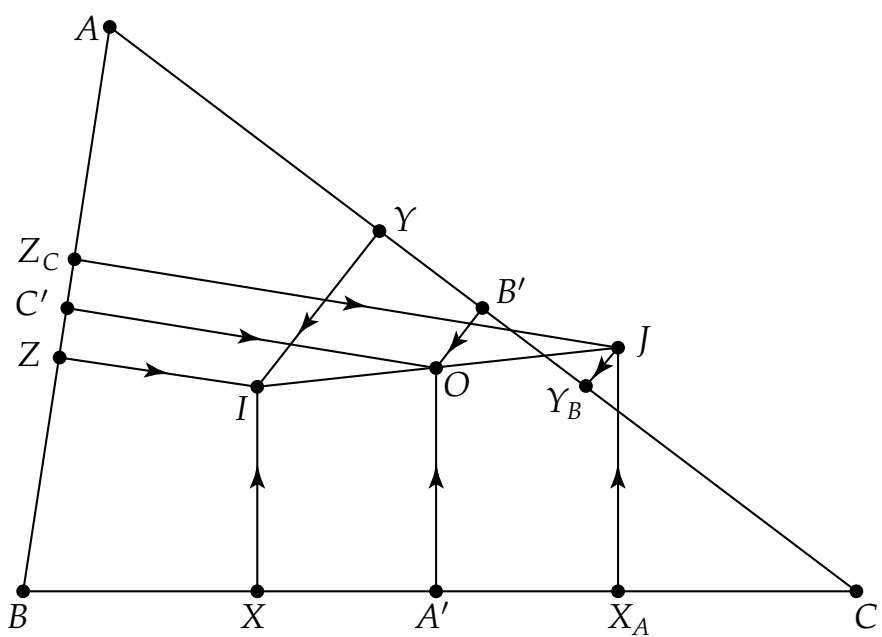

Fig. 2 
One sees immediately from Figure 2 that $O$ is the mid-point of $I J$. For, the foot $X_{A}$ of the perpendicular from $J$ to the side $B C$ is simply the point of contact of the excircle center $I_{A}$ with the side $B C$. Similarly the foot of the perpendicular from $I$ to $B C$ is the point of contact $X$ of the incircle with $B C$. But $X$ and $X_{A}$ are equidistant from the mid-point $A^{\prime}$ of $B C$, which is the foot of the perpendicular from the circumcenter $O$. Similarly for $Y$ and $Y_{B}$, and $Z$ and $Z_{C}$. Hence the result.

We see that $J$ in a sense "counterbalances" the incenter $I$ with respect to the circumcenter $O$.

The position of $J$ relative to the other central points is clarified in Figure 3, where the Euler and Nagel lines have been artificially made more symmetric. Note that as $O$ is the mid-point of both $I J$ and of $H L$, the figure $J L I H$ must be a parallelogram, hence $J L$ is parallel to $H I$. But we previously saw that $N L$ is parallel to $H I$. Therefore $J$ lies on $L H$. Moreover $J L$ equals $H I$. But we saw also that $N L=2 H I$. Therefore

$$
J \text { is the mid-point of } N L \text {. }
$$

Incidentally $J$ is also the circumcenter of the triangle $I_{A} I_{B} I_{C}$; see [1, Section 231].

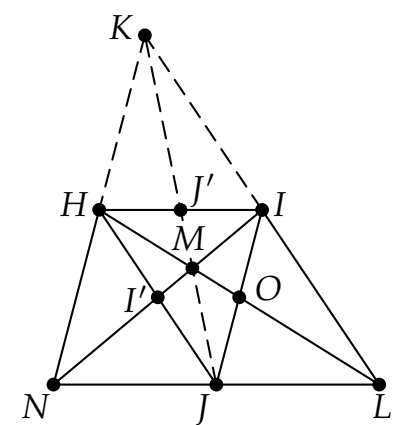

Fig. 3 Schematic diagram, showing collinearities.

\section{Completion of the scheme}

We can now complete the figure in a remarkable way (see Figure 3). Let us extend the lines $N H$ and $L I$ so as to meet in a point $K$. Since $H I$ is parallel to $N L$, the triangles $K H I$ and $K N L$ are similar. But $N L=2 H I$, therefore $K N=2 K H$ and $K L=2 K I$. In other words, $H$ and $I$ are the mid-points of $K N$ and $K L$ respectively. Hence

$$
M \text { is the median point of the triangle } K L N \text {. }
$$

From which it follows further that

$$
J, K \text { and } M \text { are collinear }
$$

and

$$
K M=2 M J .
$$

We can state also the following theorem concerning the well-established centers $H, I$, $J, L, M$ and $N$ :

$$
N H, J M \text { and } L I \text { are concurrent. }
$$


This focuses attention on the point of concurrence $K$ which, so far as is known to the author, has not been closely examined in the literature.

$K$ is itself a central point, though not a simple one, of the triangle $A B C$. The triangle $A_{1} B_{1} C_{1}$ formed by the parallels to the sides of $A B C$ through the opposite vertices is called the anticomplimentary triangle to $A B C$. Clearly $A_{1} B_{1} C_{1}$ is in a $2: 1$ homotheticity with $A B C$, center $M$. Since in Figure $3 J K=2 M J$ we see that

$$
K \text { is the countercenter of the anticomplimentary triangle. }
$$

Note also that the point $J^{\prime}$ which is the mid-point of $H I$ is the countercenter of the median triangle $A^{\prime} B^{\prime} C^{\prime}$.

We see then that the ten points

$$
H, I, J, K, L, M, N, O, I^{\prime} \text { and } J^{\prime}
$$

form a family. The ratios of parallel segments are all rational numbers. This basic scheme we may call the rational family of triangle centers.

The nine-point center $O^{\prime}$, which is the meet of $I^{\prime} J^{\prime}$ with the Euler line $H M O L$, can also be considered as a member of this family.

Hofstadter [2] has proposed a somewhat different scheme that includes $H, I, M, O, I^{\prime}$ and also $O^{\prime}$, the circumcenter of $A^{\prime} B^{\prime} C^{\prime}$, but excludes $J$ and $L$. His scheme is completed by extending $H I$ and $N O$ to meet in a point $T$. However, $T$ is not a central point of the triangle $A B C$.

\section{Coordinates for $K$}

From Figure 3 we can write down the following simple relations:

$$
K+N=2 H, \quad K+L=2 I, \quad K+2 J=3 M,
$$

also

$$
I+J=2 O, \quad H+L=2 O, \quad N+2 I=3 M .
$$

So to express the coordinates of $K$ in terms of those of $O, I$ and $M$ we have

$$
K=3 M-2 J=3 M-2(2 O-I)=2 I-4 O+3 M .
$$

The trilinear coordinates of $I, O$ and $M$ are as follows:

$$
\left.\begin{array}{rl}
I & =r(1,1,1) \\
O & =R(\cos A, \cos B, \cos C) \\
M & =\frac{2 \Delta}{3}\left(\frac{1}{a}, \frac{1}{b}, \frac{1}{c}\right)
\end{array}\right\}
$$

where $r$ is the inradius, $R$ the circumradius and $\Delta$ the area of the triangle $A B C$, and $a$, $b, c$ the lengths of the sides. Making use of the well-known relations

$$
\left.\begin{array}{rl}
\Delta & =a b c / 4 R \\
(a, b, c) & =2 R(\sin A, \sin B, \sin C)
\end{array}\right\}
$$


and also (see [5])

$$
r / R=\cos A+\cos B+\cos C-1
$$

we deduce that $K=(\xi, \eta, \zeta)$ where

$$
\xi=2 R(\sin B \sin C+\cos B+\cos C-\cos A-1)
$$

and similarly for $\eta$ and $\zeta$.

Since $\cos A=-\cos (B+C)$ we can also write

$$
\begin{aligned}
\xi & =2 R[\cos B \cos C+\cos B+\cos C-1] \\
& =2 R[(\cos B+1)(\cos C+1)-2] \\
& =4 R\left(2 \cos ^{2} \frac{1}{2} B \cos ^{2} \frac{1}{2} C-1\right)
\end{aligned}
$$

and similarly for $\eta$ and $\zeta$.

\section{The Gergonne point and related centers}

One of the simpler centers of a triangle $A B C$ is the Gergonne point $G$, defined as the meet of the three lines joining the vertices $A, B, C$ to the corresponding points of contact $X, Y, Z$ of the incircle with the three sides; see Figure 2. Kimberling [3] found by computer that $G$ lies on $I L$, and this was also discovered, and proved rigorously, by the present author [4]. From Figure 4 it is clear that by the $1: 2$ homotheticity center $M$, the Gergonne point $G^{\prime}$ of the median triangle $A^{\prime} B^{\prime} C^{\prime}$ must lie on $H J$. Thus $G^{\prime}, I^{\prime}$ and $H$ are collinear, as was pointed out in [4].

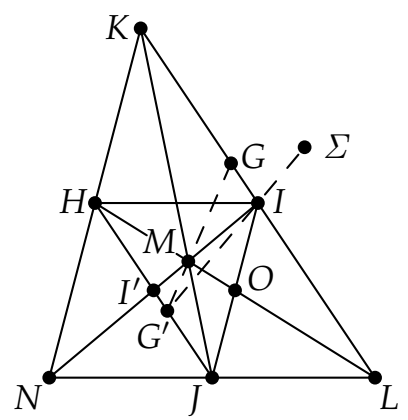

Fig. 4

Consider on the other hand the "symmedian point" $\Sigma$ of the triangle $A B C$, that is to say the point of concurrence of the three symmedians, which are the lines obtained by reflecting the lines $A A^{\prime}, B B^{\prime}, C C^{\prime}$ in $A I, B I, C I$ respectively. It is known [3] that $\Sigma$ is collinear with $I$ and the symmedian point of the excentral triangle $I_{A} I_{B} I_{C}$, which we denote by $W$. The latter point is Kimberling's $X_{9}$ and is known to lie on both $M G$ and on $H I^{\prime}$ (see [3, p. 173]). Hence $W$ coincides with $G^{\prime}$. In other words,

$G^{\prime}$ is the symmedian point of the excentral triangle $I_{A} I_{B} I_{C}$.

Thus the Gergonne point $G$ is, through $G^{\prime}$ and $\Sigma$, intimately connected with the Lemoine geometry of the triangle $A B C$; see [1, pp. 252-274]. 


\section{4-fold points of concurrence}

Just as the countercenter $J$ can be constructed from the four tritangent circles (centers $I$, $I_{A}, I_{B}$ and $I_{C}$ ) by specialising one center $I$, so we can construct three other countercenters $J_{A}, J_{B}$ and $J_{C}$, by specialising each of $I_{A}, I_{B}$ and $I_{C}$. Thus we may define $J_{A}$ as the meet of the three perpendiculars, the first from $I$ to the side $B C$, the second from $I_{B}$ to $A B$ and the third from $I_{C}$ to $A C$. Corresponding definitions apply to $J_{B}$ and $J_{C}$. Then it may easily be seen that $O$ is also the mid-point of $I_{A} J_{A}, I_{B} J_{B}$ and $I_{C} J_{C}$. Thus $O$ is the point of concurrence of the four lines $I J, I_{A} J_{A}, I_{B} J_{B}$ and $I_{C} J_{C}$. The Figure $J J_{A} J_{B} J_{C}$ is the reflexion of $I I_{A} I_{B} I_{C}$ in the point $O$.

It has been seen previously [4] that two other points on the Euler line, namely $L$ and $M$, are each 4-fold points of concurrence. For $L$ is the meet of $G I, G_{A} I_{A}, G_{B} I_{B}$ and $G_{C} I_{C}$, while $M$ is the meet of $I N, I_{A} N_{A}, I_{B} N_{B}$ and $I_{C} N_{C}$. Is there a fourth such point lying on the Euler line?

It appears that there is, namely the nine-point center $O^{\prime}$. For, the Feuerbach point $F$, which is the point of contact of the incircle with the nine-point circle, must lie on the line $I O^{\prime}$. Hence $I F$ passes through $O^{\prime}$, see Figure 5. Also there are three analogous points $F_{A}, F_{B}$ and $F_{C}$, in which the nine-point circle touches the three excircles. Hence $I_{A} F_{A}$, $I_{B} F_{B}$ and $I_{C} F_{C}$ also pass through $O^{\prime}$.

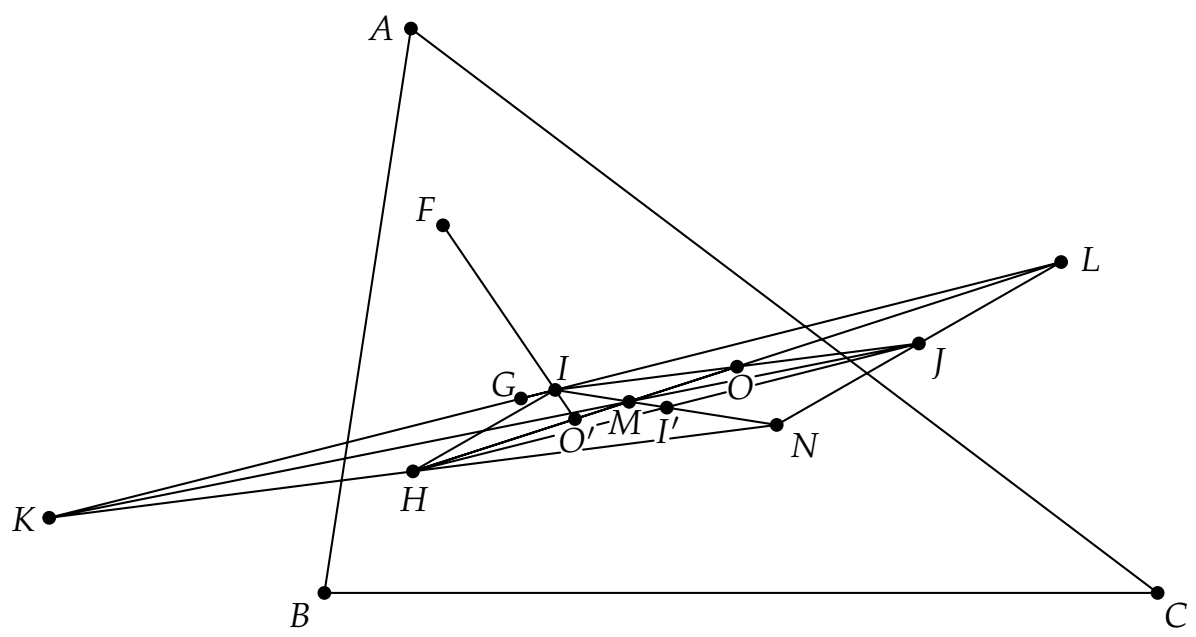

Fig. 5

Note that $F$ can be constructed independently of $O^{\prime}$; see Figure 6 . For if $\bar{X}$ is the image point of $X$ in the angle bisector $A I$ as in Figure 6 , then $F$ lies on $A^{\prime} \bar{X}$, where $A^{\prime}$ is the mid-point of $B C$ (see [1, Section 215]). Similarly $F$ lies on $B^{\prime} \bar{Y}$ and $C^{\prime} \bar{Z}$. $F$ is therefore the meet of $A^{\prime} \bar{X}, B^{\prime} \bar{Y}$ and $C^{\prime} \bar{Z}$.

Thus we have four 4-fold points of concurrence $L, O, M$ and $O^{\prime}$ all lying on the Euler line of $A B C$. The centers $I, I_{A}, I_{B}$ and $I_{C}$ are also 4-fold points of concurrence of the system. 


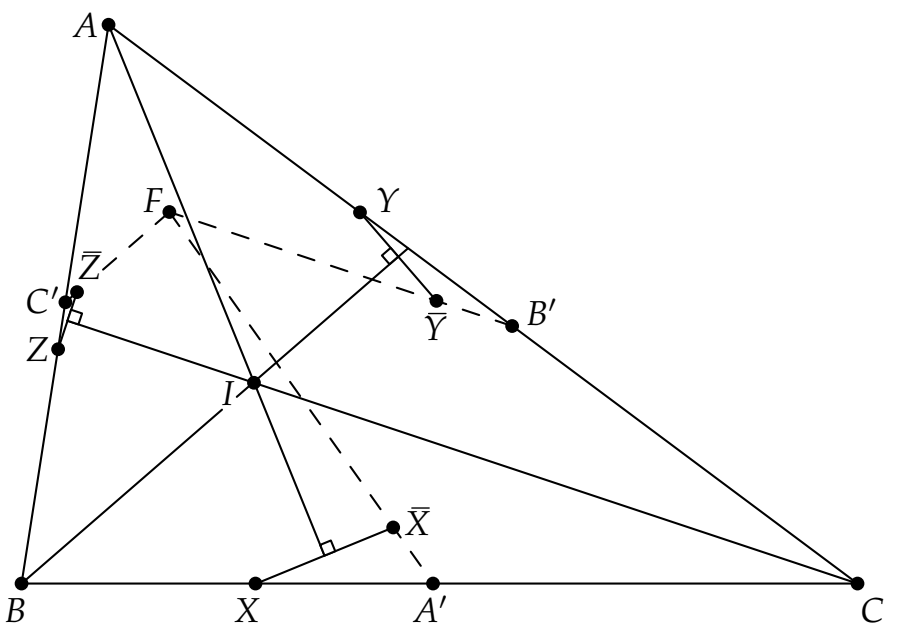

Fig. 6

Acknowledgement. I am indebted to Professor H.S.M. Coxeter for commenting on a first draft of this paper.

\section{References}

[1] Altshiller-Court, N.: College Geometry: An Introduction to the Modern Geometry of the Triangle and the Circle. (2nd ed.) Barnes Noble, New York, 1952.

[2] Hofstadter, D.R.: Discovery and dissection of a geometric gem. Geometry Turned On! Eds. J.R. King and D. Schattschneider, Math. Assoc. of America, Washington, D.C., 1997, 3-14.

[3] Kimberling, C.: Triangle Centers and Central Triangles. Congressus Numerantium 129 (1998), i-295, Winnipeg, Canada.

[4] Longuet-Higgins, M.S.: A fourfold point of concurrence lying on the Euler line of a triangle. Mathematical Intelligencer 22 (2000), 54-59.

[5] Longuet-Higgins, M.S.: On the ratio of the inradius to the circumradius of a triangle. Submitted.

Michael S. Longuet-Higgins

Institute for Nonlinear Science

University of California, San Diego

9500 Gilman Drive

La Jolla, California 92093-0402, USA

e-mail: mlonguet@ucsd.edu 